\title{
ANALYTIC FUNCTIONS WITH LARGE SETS OF FATOU POINTS
}

\section{J. S. HWANG AND PETER LAPPAN}

\begin{abstract}
For a function $f$ analytic in the unit disc $D$, and for each $\lambda>0$, let $L(\lambda)=\{z \in D:|f(z)|=\lambda\}$ denote a level set for $f$. We introduce a class $E_{1}$ of functions characterized by geometric properties of a collection of sets $\left\{L\left(\lambda_{n}\right)\right\}$, where $\left\{\lambda_{n}\right\}$ is an unbounded sequence. We show that $L_{1}$ is a proper subclass of the class $E^{2}$ of $G$. R. MacLane. Let $A_{x}$ denote the set of points $e^{i \theta}$ at which the function $f$ has $\infty$ as an asymptotic value, and let $F(f)$ denote the set of Fatou points of $f$. We prove that for a function $f$ in the class $E_{1}$, if $\Gamma$ is an arc of the unit circle such that $\Gamma \cap A_{x}=\varnothing$, then almost every point of $\Gamma$ belongs to $F(f)$.
\end{abstract}

1. Introduction. Let $D=\{z:|z|<1\}, C=\{z:|z|=1\}$, and let $f$ be a function analytic in $D$. For each real number $\lambda>0$, let $L(\lambda)=\{z \in D:|f(z)|=\lambda\}$ be a level set for $f$. Let $F(f)$ denote the set of Fatou points of $f$, that is,

$$
F(f)=\left\{e^{i \theta} \in C: f \text { has an angular limit at } e^{i \theta}\right\},
$$

and let

$$
A_{\infty}=\left\{e^{i \theta} \in C: f \text { has } \infty \text { as an asymptotic value at } e^{i \theta}\right\} .
$$

Finally, if $E$ is a subset of the closure of $D$, let $|E|$ denote the linear measure of $E$. In particular, if $\gamma$ is a curve in $D \cup C$ then $|\gamma|$ is the arc length of the curve $\gamma$.

We use the following theorem of S. Dragosh [6, Theorem 3, p. 9] as our point of departure.

THEOREM D. There exists a function $f$ analytic in $D$ such that for each arc $\Gamma$ of $C$ both $\Gamma \cap A_{\infty}=\varnothing$ and $0<|\Gamma \cap F(f)|<|\Gamma|$.

(We note that G. R. MacLane [7, Example 11, p. 75] obtained a slightly weaker result.)

In this paper, we will study the following question.

Question. What are sufficient conditions on a function $f$, analytic in $D$ to guarantee that

(S) if $\Gamma$ is an arc of $C$ with $\Gamma \cap A_{\infty}=\varnothing$ then $|F(f) \cap \Gamma|=|\Gamma|$ ?

The hypothesis that $\Gamma \cap A_{\infty}=\varnothing$ is made to exclude, for example, the modular function $M(z)$.

We introduce the class of functions $\mathscr{L}_{1}$ and we show that $\mathcal{L}_{1}$ is a subclass of the

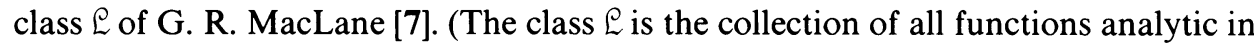

Received by the editors February 22, 1983.

1980 Mathematics Subject Classification. Primary 30D40.

Key words and phrases. Level set, Fatou point. 
$D$ such that each of the sets $L(\lambda)$ ends at points.) In our main result (Theorem 2), we show that if $f \in \mathcal{L}_{1}$ then (S) is satisfied.

To define the class $\varrho_{1}$ we need some additional notation. Throughout, the letters $n, m, j$ and $k$ are positive integers, and the letter $i$ is one of the letters " $l$ " or " $u$ ". For $\lambda>0$, let $D^{\prime}(\lambda)=\{z \in D:|f(z)|<\lambda\}$ and let $D^{u}(\lambda)=\{z \in D:|f(z)|>\lambda\}$. The notations $D_{n}^{\prime}(\lambda)$ and $D_{n}^{u}(\lambda)$ will be used to denote components of $D^{\prime}(\lambda)$ and $D^{u}(\lambda)$, respectively, and we will write $D^{i}(\lambda)=\cup_{n} D_{n}^{i}(\lambda)$ for $i \in\{l, u\}$. For a fixed $n$ and a fixed $i \in\{l, u\}$, the boundary, $\partial D_{n}^{i}(\lambda)$, of $D_{n}^{i}(\lambda)$ consists of a union of arcs in $L(\lambda)$ together with the set $\gamma_{n}^{i}(\lambda)=\partial D_{n}^{i}(\lambda) \cap C$ (which could be the empty set). Let $\left(D_{n}^{i}(\lambda)\right)^{*}$ be the smallest simply connected region containing $D_{n}^{i}(\lambda)$. We note that each $D_{n}^{l}(\lambda)$ is already simply connected, so $D_{n}^{l}(\lambda)=\left(D_{n}^{l}(\lambda)\right)^{*}$. The region $\left(D_{n}^{u}(\lambda)\right)^{*}$ is simply the region $D_{n}^{u}(\lambda)$ together with all the "holes" in this region.

We say that the number $\lambda>0$ is an admissible value for $f$ if the following are satisfied:

(1) If $\gamma_{n}^{i}(\lambda) \neq \varnothing$, then $\left|\partial\left(D_{n}^{i}(\lambda)\right)^{*}\right|<\infty$, where $i \in\{l, u\}$.

(2) If $\left\{n_{j}\right\}$ is a sequence of positive integers such that $n_{j} \rightarrow \infty$ and $\gamma_{n_{j}}^{\prime}(\lambda)=\varnothing$ for each $j$, then diam $D_{n}^{l}(\lambda) \rightarrow 0$.

(3) $\Sigma_{n}\left|\gamma_{n}^{l}(\lambda)\right|+\Sigma_{n}\left|\gamma_{n}^{u}(\lambda)\right|=2 \pi$.

We say that $f$ is in the class $\mathcal{L}_{1}$ if there exists a sequence $\left\{\lambda_{k}\right\}$ of admissible values for $f$ such that $\lambda_{k} \rightarrow \infty$.

In $\S 2$, we prove that $\varrho_{1}$ is a proper subclass of MacLane's class $\varrho$, and also that if $f$ is in the class $L_{1}$ then (S) is satisfied. Thus, the class $L_{1}$ gives an answer to the question we raised.

In $\S 3$, we give an example to show that the condition that $f$ be in the class $\mathcal{L}_{1}$ is not a necessary condition to give an affirmative answer to our question. In addition, we give an example of a function $f$ in the class $\mathscr{L}_{1}$ for which $A_{\infty}=C$ and $|F(f)|=0$. Finally, we show that no containment relationship exists between the class $\varrho_{1}$ and the class of normal functions.

In $\$ 4$, we give some open questions.

2. The main results. We begin with some lemmas concerning the geometry of the sets $D_{n}^{i}(\lambda)$, where $i \in\{l, u\}$ and $\lambda$ is an admissible value.

We will use several times a result due to F. and M. Riesz (see [5, Theorem 3.3, p. 50]).

THEOREM R. Let $\varphi$ be a one-to-one conformal mapping from $D$ onto a simply connected region $R$ bounded by a rectifiable closed curve $\Gamma$. Then $\varphi$ can be extended continuously to the closure of $D, \varphi^{\prime}\left(e^{i \theta}\right)$ exists for almost all $\theta \in[0,2 \pi)$, and

$$
|\Gamma|=\int_{0}^{2 \pi}\left|\varphi^{\prime}\left(e^{i \theta}\right)\right| d \theta
$$

In addition, if $E$ is a subset of $\Gamma$ then $|E|=0$ if and only if $\left|\varphi^{-1}(E)\right|=0$.

This result is normally stated for the case where $\Gamma$ is a Jordan curve. However, the proof appearing in [5, pp. 50-53] is valid for the case where $\Gamma$ is a closed rectificable curve (and not necessarily a simple closed curve). 
Lemma 1. Let $f$ be a function analytic in $D$, let $i \in\{l, u\}$, and let $\lambda>0$ be an admissible value for $f$. For each $n$ and for each point $p \in \gamma_{n}^{i}(\lambda)$, there exists a Jordan arc $\gamma_{p}$ in $D_{n}^{i}(\lambda) \cup\{p\}$ such that $p$ is an endpoint of $\gamma_{p}$.

Proof. Let $p \in \gamma_{n}^{i}(\lambda)$. Because of $(1), \partial\left(D_{n}^{i}(\lambda)\right)^{*}$ is a rectifiable closed curve. Let $\varphi$ be a conformal mapping from $D$ onto $\left(D_{n}^{i}(\lambda)\right)^{*}$. By Theorem R, $\varphi$ can be extended continuously to the closure of $D$. Thus, there exists a radius $R_{p}$ of $D$ such that $\varphi\left(R_{p}\right)=\gamma_{p}^{\prime}$ is an arc in $\left(D_{n}^{i}(\lambda)\right)^{*}$ ending at $p$.

If $\gamma_{p}^{\prime} \subset D_{n}^{i}(\lambda) \cup\{p\}$, then $\gamma_{p}=\gamma_{p}^{\prime}$ is the desired curve. However, if $\gamma_{p}$ meets one or more "holes" in $D_{n}^{i}(\lambda)$, then we can construct a sequence of detours around these "holes". Further, because of (2), the diameters of these detours will be small for the "holes" which are near the unit circle $C$. Thus, there exists Jordan arc $\gamma_{p^{\prime}}$ obtained from $\gamma_{p}^{\prime}$ by means of appropriate detours around the "holes" in $D_{n}^{i}(\lambda)$, so that $\gamma_{p} \subset D_{n}^{i}(\lambda) \cup\{p\}$ and $\gamma_{p}$ has $p$ as an endpoint. (The formal construction of these detours is a standard elementary exercise, so we omit the details.)

LEMMA 2. Let $f$ be a function analytic in $D$ and let $\lambda>0$ be an admissible value for $f$. Let $E(\lambda)=\left\{p \in C: p \in \gamma_{n}^{i}(\lambda) \cap \gamma_{m}^{i^{\prime}}(\lambda)\right.$, where either $i \neq i^{\prime}$ or $n \neq m$ and $i, i^{\prime} \in$ $\{u, l\}\}$. Then $E(\lambda)$ is a countable set.

Proof. Define a function $g$ on $D$ such that $g(z)=n$ for $z \in D_{n}^{l}(\lambda), g(z)=-n$ for $z \in D_{n}^{u}(\lambda)$, and $g(z)=0$ for $z \in L(\lambda)$. If $p \in E(\lambda)$, then by Lemma 1 we have that $p$ is an ambiguous point for the function $g$, and by the Bagemihl Ambiguous Point Theorem [2] we have that $E(\lambda)$ is a countable set.

We are now in a position to prove that the class $\mathcal{L}_{1}$ is a subclass of $\mathcal{L}$. We write $f \in \mathscr{L}_{1}(\mathcal{L})$ to denote that $f$ is in the class $\mathscr{L}_{1}(\mathcal{L})$.

THEOREM 1. If $f \in \mathcal{L}_{1}$ then $f \in \mathcal{L}$.

Proof. Suppose $f \in \mathcal{L}_{1}$ and let $\lambda>0$. We need to show that $L(\lambda)$ ends at points of $C$. Suppose that this is not the case. Then there exists a sequence of arcs $\left\{\gamma_{n}\right\}$ in $L(\lambda)$ and a number $\varepsilon_{0}>0$ such that for each $n$ we have that $\gamma_{n} \subset\{z:|z|>1-$ $(1 / n)\}$ and the diameter of $\gamma_{n}$ exceeds $\varepsilon_{0}$. Further, we may assume that there exists an $\operatorname{arc} \Gamma$ of $C$ with $|\Gamma|>\varepsilon_{0} / 2$ such that for each point $p \in \Gamma$ and each Jordan arc $\gamma_{p}$ in $D \cup\{p\}$ with endpoint at $p$ we have $\gamma_{p} \cap \gamma_{n} \neq \varnothing$ for all but a finite number of $n$. Let $\lambda_{0}$ be an admissible value for $f$ such that $\lambda_{0}>\lambda$. We now have two possibilities: (a) the arcs $\gamma_{n}$ occur in infinitely many different components of $D^{l}\left(\lambda_{0}\right)$, or (b) all but a finite number of the arcs $\gamma_{n}$ appear in a single component of $D^{\prime}\left(\lambda_{0}\right)$.

Suppose that (a) happens. By renumbering, if necessary, we may assume that $\gamma_{n} \subset D_{n}^{l}\left(\lambda_{0}\right)$ for each $n$. By (3), we have for almost all $p \in \Gamma$ that $p \in \gamma_{n_{p}}^{i}\left(\lambda_{0}\right)$ for some integer $n_{p}$ and $i \in\{l, u\}$. Thus, for $p \in \gamma_{n_{p}}^{i}\left(\lambda_{0}\right)$ we have from Lemma 1 that there exists a Jordan arc $\gamma_{p} \subset D_{n_{p}}^{i}\left(\lambda_{0}\right) \cup\{p\}$ having $p$ as an endpoint. However, for almost all $p \in \Gamma$ we have $\gamma_{p} \cap \gamma_{n} \neq \varnothing$ for all but a finite number of $n$. Hence no single $D_{n_{p}}^{i}\left(\lambda_{0}\right)$ can be found to contain such a $\gamma_{p}$. It follows that case (a) cannot occur.

If case (b) occurs, then there exists a single integer $n_{0}$ such that $\gamma_{n} \subset D_{n_{0}}^{l}\left(\lambda_{0}\right)$ for all but a finite number of $n$. Thus $\Gamma \subset \gamma_{n_{0}}^{l}\left(\lambda_{0}\right)$. Let $\varphi$ be a conformal mapping from 
$D$ onto $D_{n_{0}}^{l}\left(\lambda_{0}\right)$. Then, by (1) and Theorem R, the set $\varphi^{-1}(\Gamma)$ is a set of positive measure on $C$. But the function $g(t)=f(\varphi(t))$ is a bounded analytic function in $D$ and the sets $\left\{\varphi^{-1}\left(\gamma_{n}\right)\right\}$ are all connected subsets of $L(\lambda)$ for the function $g$, and hence $\lim \sup _{n \rightarrow \infty} \operatorname{diam} \varphi^{-1}\left(\gamma_{n}\right) \geqslant\left|\varphi^{-1}(\Gamma)\right|>0$, which means that $g$ is not in the class $\mathcal{L}$ (see [7]). It follows the case (b) cannot occur either, and so we must have $f \in \mathcal{L}$.

We can now give an answer to our question.

THEOREM 2. If $f \in \mathcal{L}_{1}$ and if $\Gamma$ is a subarc of $C$ such that $A_{\infty} \cap \Gamma=\varnothing$, then $|F(f) \cap \Gamma|=|\Gamma|$.

Proof. By a theorem of Plessner (see [5, Theorem 8.2, p. 147]) almost every point of $C$ is either a Fatou point or a Plessner point, that is, a point at which each angular cluster set is the extended complex plane. We will show that if $f \in \varrho_{1}$ and if $p$ is a Plessner point which is not a member of a fixed set $H$, where $|H|=0$, then $p \in A_{\infty}$. This will establish the theorem.

Let $f \in \mathcal{L}_{1}$ and let $\left\{\lambda_{k}\right\}$ be a sequence of admissible values for $f$ such that $\lambda_{k} \rightarrow \infty$. If $H_{n}^{i}\left(\lambda_{k}\right)=\left\{p \in \gamma_{n}^{i}\left(\lambda_{k}\right)\right.$ : the tangent to $\partial\left(D_{n}^{i}\left(\lambda_{k}\right)\right)^{*}$ at $p$ either does not exist or is not equal to the tangent to $C$ at $p\}$, then $\left|H_{n}^{i}\left(\lambda_{k}\right)\right|=0$ by Theorem R. Hence

$$
H^{\prime}=\bigcup_{k}\left(\bigcup_{n} H_{n}^{\prime}\left(\lambda_{k}\right) \cup \bigcup_{m} H_{m}^{u}\left(\lambda_{k}\right)\right)
$$

satisfies $\left|H^{\prime}\right|=0$ and, if

$$
H=H^{\prime} \cup \bigcup_{k}\left(C-\left(\bigcup_{n} \gamma_{n}^{l}\left(\lambda_{k}\right) \cup \bigcup_{m} \gamma_{m}^{u}\left(\lambda_{k}\right)\right)\right),
$$

then $|H|=0$. If $p \notin H$, we have that for each $k$ there exists an integer $n_{k}$ and $i_{k} \in\{l, u\}$ such that $p \in \gamma_{n_{k}}^{i_{k}}\left(\lambda_{k}\right)$ and the tangent to $\partial\left(D_{n_{k}}^{i_{k}}\left(\lambda_{k}\right)\right)^{*}$ at $p$ coincides with the tangent to $C$ at $p$. If $i_{k} \stackrel{=}{=}$, then each angular cluster set is bounded, so $p$ cannot be a Plessner point. Thus, if $p$ is a Plessner point, then $i_{k}=u$ for each $k$. In this case, $p \in \gamma_{n_{k}}^{u}\left(\lambda_{k}\right)$, so by Lemma 1 there exists a Jordan $\operatorname{arc} \gamma_{p, k}$ in $D_{n_{k}}^{u}\left(\lambda_{k}\right) \cup\{p\}$ having $p$ as an endpoint. By renumbering, if necessary, we may assume that $D_{n_{k+1}}^{u}\left(\lambda_{k+1}\right) \subset$ $D_{n_{k}}^{u}\left(\lambda_{k}\right)$ for each $k$. Both $\gamma_{p, k}$ and $\gamma_{p, k+1}$ are the subsets of $D_{n_{k}}^{u}\left(\lambda_{k}\right)$, so we can cut off $\gamma_{p, k}$ at some appropriate point $w_{k}^{*}$ and delete the portion of $\gamma_{p, k}$ from $w_{k}^{*}$ to $p$, join $w_{k}^{*}$ to a point $w_{k+1} \in \gamma_{p, k+1}$ by a Jordan arc $\gamma_{k}$ in $D_{n_{k}}^{u}\left(\lambda_{k}\right)$, and delete the component of $\gamma_{p, k+1}-\left\{w_{k+1}\right\}$ which does not end at $p$. If we do this for each $k$, we get a Jordan arc $\gamma$, consisting of $\cup_{k} \gamma_{k}$ and appropriate portions of the arcs $\gamma_{p, k}$ between $w_{k}$ and $w_{k}^{*}$, such that $f(z) \rightarrow \infty$ as $z \rightarrow p$ along $\gamma$. (We need to be sure that the points $w_{k}$ are chosen so that the diameter of the component of $\gamma_{p, k}-\left\{w_{k}\right\}$ having $p$ as an endpoint goes to 0 as $k \rightarrow \infty$, and also that diam $\gamma_{k} \rightarrow 0$ as $k \rightarrow \infty$. The former can be accomplished by choosing the $w_{k}$ appropriately, while the latter can be accomplished by carefully deforming the line segment from $w_{k}^{*}$ to $w_{k+1}$ to a curve $\gamma_{k}$, for condition (2) guarantees that an appropriate deformation is possible.) Thus $p \in A_{\infty}$ and the proof is complete.

We remark that the function described in Theorem $D$ is in the class $\varrho$ but it violates the conclusion of Theorem 2 , so this function is not in the class $E_{1}$. But $e_{1} \subset \mathcal{E}$ by Theorem 1 , so the containment is proper. 
3. Examples. We now show that there exists a function which satisfies the conclusion of Theorem 2 but is not a member of $\mathcal{L}_{1}$.

EXAMPLE 1. There exists a function $f \in \mathcal{L}$ such that $f \notin \mathcal{L}_{1}$, but for each arc $\Gamma$ in $C$ for which $\Gamma \cap A_{\infty}=\varnothing$ we have $|\Gamma|=|\Gamma \cap F(f)|$.

Proof. Let $P=\{z=x+i y: 0 \leqslant x<1, y=0\}$ and for each positive integer $n$ let

$$
H_{n}=\{z=x+i y: x=2 n /(2 n+1),|y| \leqslant 1 /(2 n+1)\},
$$

and let $H=P \cup \cup_{n=1}^{\infty} H_{n}$. For each positive integer $n$, let

$$
K_{n}=\{z=x+i y: x=(2 n-1) /(2 n), 1 /(4 n+2) \leqslant|y| \leqslant \sqrt{4 n-1} /(2 n)\},
$$

and let $K=\cup_{n=1}^{\infty} K_{n}$. By a theorem of Arakelian [1] (see also [4]), there exists a function $f$ analytic in $\{z:|z+1|<2\}$ such that $f(z) \rightarrow 0$ as $z \rightarrow 1$ on $H$ and $|f(z)-n|<1 / n$ for $z \in K_{n}$ for each $n$. Let $\lambda>\operatorname{supp}\{|f(z)|: z \in H\}$, and restrict $f$ to the unit disc $D$. Then the component $D_{n_{0}}^{l}(\lambda)$ of $D^{l}(\lambda)$ for which $H \subset D_{n_{0}}^{l}(\lambda)$ satisfies $(2 n) /(2 n+1)+i /(2 n+1) \in D_{n_{0}}^{l}(\lambda)$ and $(2 n-1) /(2 n)+i /(4 n+2) \notin$ $D_{n_{p}}^{l}(\lambda)$ for $n$ sufficiently large. It follows that $\left|\partial D_{n_{0}}^{l}(\lambda)\right|=\infty$. Also, $1 \in \gamma_{n_{0}}^{l}(\lambda)$ and thus no large $\lambda$ is an admissible value for $f$, since (1) is violated for all large $\lambda$. Thus $f \notin \mathcal{L}_{1}$, but $\{z:|z|=1, z \neq 1\} \subset\{z:|z+1|<2\}$, so $f$ is analytic at each point of $C-\{1\}, C-\{1\} \subset F(f)$ and $A_{\infty} \subset\{1\}$. (It is not clear whether $A_{\infty}=\varnothing$ or $\left.A_{\infty}=\{1\}.\right)$ Therefore, $|\Gamma|=|\Gamma \cap F(f)|$ for each arc $\Gamma$ in $C$.

From this example we see that Theorem 2 does not give a unique answer to the question posed in the introduction.

ExAmple 2. There exists a function $f \in \mathcal{L}_{1}$ such that $A_{\infty}$ is dense in $C$ and $|F(f)|=0$.

Proof. Let $f(z)=\prod_{k=1}^{\infty}\left\{1-\left(z /\left(1-n_{k}^{-1}\right)\right)^{n_{k}}\right\}$ where $\left(n_{k+1} / n_{k}\right) \rightarrow \infty$ as $k \rightarrow \infty$. By a result of Bagemihl, Erdös and Seidel [3], we have that $A_{\infty}=C$ and, for each $\lambda>0$, each of the sets $D_{n}^{l}(\lambda)$ is a relatively compact subset of $D$ with diam $D_{n}^{l}(\lambda) \rightarrow 0$ as $n \rightarrow \infty$. Hence $D^{u}(\lambda)$ consists of a single component and $\gamma^{u}(\lambda)=C$ for each $\lambda>0$. Thus $\left(D^{u}(\lambda)\right)^{*}=D$ and thus each $\lambda>0$ is an admissible value for $f$, so $f \in \mathcal{L}_{1}$. But $\infty$ is the only possible Fatou value of $f$ and hence $|F(f)|=0$ by a theorem of Privalov (see [5, Corollary 1, p. 146]).

We note that the function in Example 2 is not a normal function but it is in $\mathcal{L}_{1}$, while the function given in Theorem $\mathrm{D}$ is a normal function which is not in the class $\mathcal{E}_{1}$. Thus, there does not exist a containment relationship between the class $\mathscr{L}_{1}$ and the class of normal analytic functions.

4. Open questions. Define the class $\mathcal{L}_{2}$ to be the collection of all functions $f$ analytic in $D$ such that for each arc $\Gamma$ in $C$ for which $\Gamma \cap A_{\infty}=\varnothing$ it is true that $|\Gamma \cap F(f)|=|\Gamma|$. In view of Theorems 1, 2 and D, Example 1 and results in [7], we must have $\mathcal{L}_{1} \subseteq \mathcal{L}_{2} \subseteq \mathcal{L}$. The following is a natural question.

Question 1. Can the class $\varrho_{2}$ be characterized in terms of level sets $L(\lambda)$ and/or the sets $D^{l}(\lambda)$ and $D^{u}(\lambda)$ ?

One possible approach to this question would be to look for an appropriate modification of condition (1) while retaining (2) and (3) in the consideration of the 
admissible values. We do not have a specific candidate for a modified condition (1) at this time.

Another question is suggested by Example 2.

Question 2. Does there exist a function $f \in \mathcal{L}_{1}$ such that $A_{\infty}$ is a countable dense subset of $C$ and $|F(f)|=0$ ?

A natural candidate to answer this question affirmatively would be the elliptic modular function, which we denote by $M(z)$. Unfortunately, $M \notin \mathcal{L}_{1}$. To see this, we note that for each $\lambda>1$ it is the case that each component $D_{n}^{u}(\lambda)$ satisfies the property that $\gamma_{n}^{u}(\lambda)$ is a single point of $C$ (which is a point in $A_{\infty}$ ). Thus $D^{\prime}(\lambda)$ consists of a single component, and $\left|\gamma^{\prime}(\lambda)\right|=2 \pi$. Let $\varphi$ be a conformal mapping from $D$ onto $D^{\prime}(\lambda)$. Then $g(z)=M(\varphi(z))$ is a bounded analytic function, so $|F(g)|=2 \pi$. If $\left|\partial D^{l}(\lambda)\right|<\infty$, then $\varphi$ can be extended continuously to the closure of $D$, and $\left|\varphi^{-1}\left(\gamma^{l}(\lambda)-A_{\infty}\right)\right|>0$ by Theorem R. At any point of $\gamma^{l}(\lambda)-A_{\infty}$ the function $M$ has only 0 and 1 as possible asymptotic values, and thus the function $g$ can have only 0 and 1 as asymptotic values on the set $\varphi^{-1}\left(\gamma^{l}(\lambda)-A_{\infty}\right)$. But this means that $\left|\varphi^{-1}\left(\gamma^{l}(\lambda)-A_{\infty}\right)\right|=0$ according to the theorem of Privalov, in violation of our previous assertion that $\left|\varphi^{-1}\left(\gamma^{l}(\lambda)-A_{\infty}\right)\right|>0$. It follows that $\left|\partial D^{\prime}(\lambda)\right|=\infty$ for $\lambda>1$, and thus $M \notin \mathcal{L}_{1}$.

\section{REFERENCES}

1. N. U. Arakelian, Uniform and tangential approximation by analytic functions, Izv. Akad. Armjan. SSR Ser. Mat. 3 (1968), 273-286. (Russian)

2. F. Bagemihl, Curvilinear cluster sets of arbitrary functions, Proc. Nat. Acad. Sci. U.S.A. 41 (1955), 379-382.

3. F. Bagemihl, P. Erdös and W. Seidel, Sur quelques propriétés frontières des fonctions holomorphes définies par certains produits dans le cercle-unité, Ann. Sci. École Norm. Sup. (3) 70 (1953), 135-147.

4. L. Brown, P. M. Gauthier and W. Seidel, Complex approximation for vector-valued functions with an application to boundary behavior, Trans. Amer. Math. Soc. 191 (1974), 149-163.

5. E. F. Collingwood and A. J. Lohwater, The theory of cluster sets, Cambridge Univ. Press, London and New York, 1966.

6. S. Dragosh, The distribution of Fatou points of bounded and normal analytic functions, Ann. Acad. Sci. Fenn. Ser. A I Math. 296 (1971), 1-11.

7. G. R. MacLane, Asymptotic values of holomorphic functions, Rice University Studies 49 (1963).

Institute of Mathematics, Academia Sinica, Taipei, Taiwan

Department of Mathematics, Michigan State University, East Lansing, Michigan 48824 